\title{
A New Way of Anemometer Calibration and Its Application to a Sonic Anemometer
}

\author{
C. KRAAN AND W. A. OOST \\ Royal Netherlands Meteorological Institute (KNMI), Division of Oceanographic Research. The Netherlands
}

(Manuscript received 17 June 1988, in final form 29 November 1988)

\section{ABSTRACT}

\begin{abstract}
To obtain accurate results from flux measurements with the eddy-correlation method, one has to be very sure about the causes of any mean vertical wind speed. Furthermore, it is generally not sufficient to use simple analytical expressions for the relation between the signals of the anemometer and the wind speed components. We therefore introduce a new calibration/interpretation method for anemometers with fixed sensors, which provides the desired extra accuracy, and then consider the results of the application of this method to eight successive calibrations of a sonic anemometer.
\end{abstract}

\section{Introduction}

The most straightforward way to measure a turbulent vertical momentum flux is the eddy-correlation method. A fast responding anemometer measures the horizontal wind component $u$ and the vertical component $w$; their product is the total vertical momentum flux. To obtain its turbulent part, the contribution of the mean wind components $U$ and $W$ must be subtracted. The relations between the measured, mean and turbulent wind speeds are

$$
u=U+u^{\prime} ; \quad w=W+w^{\prime},
$$

where $u^{\prime}$ and $w^{\prime}$ are the turbulent components of the wind. The relation between the total flux, the contribution of the mean winds and the turbulent flux is then

$$
\langle u w\rangle=U W+\left\langle u^{\prime} w^{\prime}\right\rangle,
$$

the angular brackets indicating mean values.

The traditional way to get rid of the $U W$ term is to assume that $W$ was in fact zero and that the nonzero value, which one finds most of the time, was due to misalignment of the wind sensor during the calibration, during the measurements or during both; this state of affairs is then corrected by a coordinate rotation. Some attempts have been made to do better (e.g. Jones and Smith 1978), but as far as we are aware the method is still generally used. How dangerous this is, especially over a low drag surface like the sea, can be shown by a simple and straightforward calculation:

$$
\begin{aligned}
\langle u w\rangle & =\left\langle u^{\prime} w^{\prime}\right\rangle \times\left(1+U W /\left\langle u^{\prime} w^{\prime}\right\rangle\right) \\
& =\left\langle u^{\prime} w^{\prime}\right\rangle \times\left[1-(W / U) /\left(C_{D}\right)\right],
\end{aligned}
$$

Corresponding author address: Dr. C. Kraan, Royal Netherlands Meteorological Institute (KNMI), P.O. Box 201, 3730 AE de Bilt, The Netherlands. with the drag coefficient

$$
C_{D}=-\left\langle u^{\prime} w^{\prime}\right\rangle / U^{2} .
$$

If we take, for example, an elevation of one single degree and a value of $1.2 * 10^{-3}$ for the drag coefficient at $10 \mathrm{~m}$. height (a value generally accepted for marine situations), (1) becomes

$$
\begin{aligned}
\langle u w\rangle & =\left\langle u^{\prime} w^{\prime}\right\rangle \times\left[1-0.0175 /\left(1.2 \times 10^{-3}\right)\right] \\
& =\left\langle u^{\prime} w^{\prime}\right\rangle \times(1-15) .
\end{aligned}
$$

This huge sensitivity to misalignment is caused by the influence of the $U W$ term in (1) and should not be confused with the influence of misalignment on the turbulent flux $\left\langle u^{\prime} w^{\prime}\right\rangle$ itself, which is much smaller, as we can show as follows: Indicating measured quantities with a suffix $m$, the relation between the measured wind speed components and the desired ones can be written as

$$
\begin{aligned}
u_{m} & =u \cos (\phi)+w \sin (\phi) \\
w_{m} & =-u \sin (\phi)+w \cos (\phi),
\end{aligned}
$$

where $\phi$ indicates the tilt angle and which leads to

$$
\begin{aligned}
\left\langle u^{\prime} w^{\prime}\right\rangle_{m}=\left\langle u^{\prime} w^{\prime}\right\rangle & \cos (2 \phi) \\
& -\left(\left\langle u^{\prime 2}\right\rangle-\left\langle w^{\prime 2}\right\rangle\right) \sin (2 \phi) / 2 .
\end{aligned}
$$

The ratio of $\left\langle u^{\prime} w^{\prime}\right\rangle$ and $\left\langle u^{\prime} w^{\prime}\right\rangle_{m}$ will then change with only $7 \%$ per degree of tilt if we assume (e.g., Geernaert et al. 1987) that

$$
\sigma_{u} / u_{*}=2.3 \text { and } \sigma_{w} / u_{*}=1.3 .
$$

In view of the foregoing, it is clear that one needs to be very sure that a nonzero $W$ is indeed caused by misalignment and not by any other more complicated effect. Such other effects are well known, the most prominent among them being the distortion of the flow 
by the instrument platform or mast, as discussed by, e.g., Wucknitz (1980) and Wyngaard (1981). In his article Wyngaard shows that the consequences of misalignment are not identical to those of flow distortion, which underlines how important it is to know the causes of a nonzero $W$.

We intend to show that standard data handling and a manufacturer-provided calibration cannot guarantee trustworthy values for momentum fluxes and we will provide a method to obtain the precision required for practical use of the data. For a sonic anemometer, a commonly used instrument for this type of measurements, the problem lies even deeper and one can run into serious troubles if the calibration of the instrument is not meticulously performed and repeated at regular intervals.

The problem has been noted before (Wyngaard and Zhang 1985) but was attributed there mainly to transducer shadow effects. Wyngaard and Zhang state that the disturbances should be minimal for our type of sensor array, although they admit that the deviations from the ideal response are caused by a complex interaction between the sensor head and the flow. The type of calibration we propose takes care of all effects, at least under wind tunnel conditions. We have to admit that in principle, because the flow conditions in the field are not the same as those in the wind tunnel, differences could exist between the fluid dynamical properties of the sonic in the two situations. We do not expect, though, that these differences will be noteworthy.

It should be stressed that all this does not mean that the sonic anemometer in general, or our specimen in particular, is a bad instrument; other instruments may and will have the same or even more serious problems.

\section{The need for a better calibration method}

In the course of the development of the pressure anemometer [a new type of anemometer intended for use in adverse circumstances, such as exist close to the sea surface (Oost 1983)], we had to devise a new calibration method, as the anemometer turned out to have a rather complicated direction sensitivity. In this method the instrument is put in a wind tunnel on a frame which can be rotated in small steps around a horizontal (elevation) and a vertical (azimuth) axis.

When we acquired a sonic anemometer (a Kayo Denki DAT-300) with the well-known nonorthogonal sensor arrangement (Fox 1968) and electronic conversion to Cartesian coordinates, we applied the method to this instrument as well, as a check. This required only minor adaptations relative to the procedure for the pressure anemometer.

The end product of such a calibration is a set of tables, linking the real values of wind speed, azimuth and elevation, derived from the wind tunnei gauge and the orientation of the calibration frame, to what we call the apparent values, viz., those calculated by assuming a certain wind speed dependence and directional sensitivity of the instrument (linear and a cosine function, respectively, in the case of a sonic anemometer).

Figure 1 is a combined graph of two of these tables in which the real values for azimuth and elevation, indicated by ' $X$ ', and the apparent values, indicated by ' + ', have been plotted. Ideally both groups of symbols should coincide. It is clear from the figure that deviations of $1^{\circ}$ and more do occur, both for elevation and for azimuth, especially if the wind comes from an offaxis direction. In view of the example in the Introduction, this shows that the assumed cosine relation is not good enough if one wishes to use the anemometer for flux measurements. We therefore propose the calibration/interpretation procedure described below.

\section{The calibration procedure}

The wind tunnel in which the calibration takes place must have a measuring section sufficiently large to rotate the instrument on the frame over the desired azimuth and elevation ranges without a loss of accuracy due to inhomogeneity of the flow field. A calibration consists of two parts: a wind speed and a direction calibration, the latter one consisting of three separate runs.

The wind speed calibration provides the relation between the airspeed in the tunnel and the signal of one sensor of the anemometer, which we will indicate as component $A$. During this part of the calibration procedure the anemometer is oriented so as to maximize the signal of $A$. The wind tunnel speed is then varied in steps over the full calibration range, while the instrument retains its orientation. The lower end of this range is determined by the low wind speed properties of the wind tunnel and the range of its speed sensor; for the wind tunnel we commonly use it is not advisable to go below about $4 \mathrm{~m} \mathrm{~s}^{-1}$. The high wind speed end is determined by the maximum speed of the wind tunnel, which is in our case about $25 \mathrm{~m} \mathrm{~s}^{-1}$. At every wind speed step readings of about half a minute duration are taken of the sensor signal and the airspeed gauge of the tunnel.

The direction calibration enables us to derive the wind direction from the ratios of the instrument signals. During each of the three runs the wind speed is kept constant and the instrument is rotated in $5^{\circ}$ steps over the desired azimuth and elevation ranges. For our sonic we use an azimuth range from $-60^{\circ}$ to $+60^{\circ}$ and an elevation range from $-45^{\circ}$ to $+45^{\circ}$, relative to the central axis. The latter range is taken rather spaciously to prevent that we should meet elevations outside the calibration range during measurements in, e.g., an unstable, low wind speed situation. The step size is chosen as large as possible, but small enough to be able to use linear interpolations without introducing significant errors. At every step the three sensor signals, the read- 


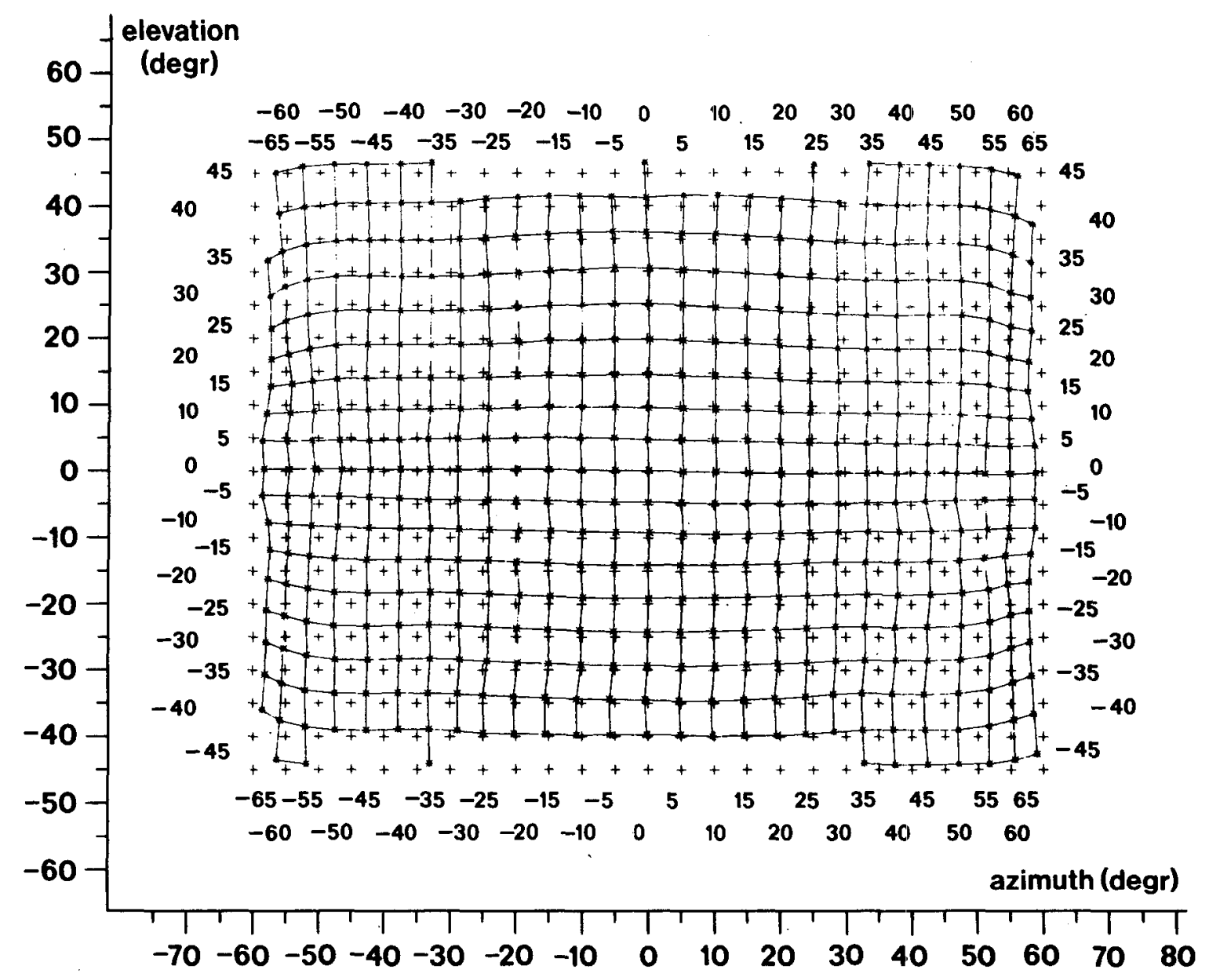

FiG. 1. The relation between the real and apparent azimuth and elevation for a sonic anemometer. (For the meaning of the words "real" and "apparent" see text.) The figure is valid for one wind speed $\left(10.2 \mathrm{~m} \mathrm{~s}^{-1}\right)$. The "+" signs are the grid points of the mesh of apparent angles. The real angles belonging to these grid points are depicted by the " $X$ " signs. Real angles belonging to the same (apparent) azimuth or elevation have been connected by lines. At the end points of these lines the pertinent values of the apparent angles are indicated to show the connection with the real angles. In the case of a pure cosine direction dependence the two sets of symbols should coincide.

ings of the orientation sensors of the calibration frame and the tunnel speed are registered, each step lasting a few seconds. To accommodate changes due to Reynolds effects, we perform the direction calibration for three wind speeds, selected to give optimal coverage of the full wind speed range.

\section{Processing of calibration data}

Initially, after preprocessing (cleaning and reformatting of data tapes, correction for signal levels at wind speed zero and averaging of data belonging to the same calibration point), the wind speed calibration and the three direction calibrations are treated separately.
From the wind speed calibration data we calculate the coefficients for a linear relation between the signal of component $A$ and the wind speed, using an unweighted least-squares method. The resulting relation can be given in general as

$$
v=f\left(s_{A}\right),
$$

with $v$ indicating the wind speed and $s_{A}$ the signal of component $A$.

The treatment of the direction calibration runs is more complicated. For each run we first retrieve the signal of component $A$ in the situation that the instrument had the same orientation as during the wind speed calibration $\left(s_{A}\right)$. We then use the following data at each calibration point: 
1) The ratio of $s_{m}$, the strongest of the three anemometer signals, and $s_{A}$; we call this ratio the speed index, $i_{s}$. This quantity will be used to calculate the wind speed.

2) The azimuth and elevation of the wind, as recorded from the position sensors of the calibration frame (the real azimuth and elevation).

3) The azimuth and elevation calculated from the three anemometer signals, assuming a cosine direction dependence (the apparent azimuth and elevation).

The pretreatment of the data provides us with a translate table, in which the values of the apparent azimuth $a_{\text {app }}$, the apparent elevation $e_{\text {app }}$ and the speed index $i_{s}$ are given for all points of the grid of real angles (real azimuth $a_{r}$ and real elevation $e_{r}$ ). This table can be noted as

$$
\left(a_{\mathrm{app}}, e_{\mathrm{app}}, i_{s} \mid a_{r}, e_{r}\right) \text {. }
$$

From measured data, however, one cannot calculate the real, but only the apparent values, using the assumed direction dependence. To apply our calibration, we need the real values as a function of the apparent values. Therefore the next step in the treatment of a direction calibration run is an inversion of the table, leading to one in which the real azimuth, the real elevation and the speed index are given for all points of a grid of apparent angles. For this new grid we use the same type of grid as for the real angles. In our notation:

$$
\left(a_{r}, e_{r}, i_{s} \mid a_{\mathrm{app}}, e_{\mathrm{app}}\right) \text {. }
$$

We end up with three of these tables, each one valid only at the wind speed at which it was obtained.

To get the direction calibration for all wind speeds within the calibration range, the tables must now be combined. We do this by connecting by a parabola the values for a quantity at the same point of the grid of apparent values in each of the three tables. The procedure is applied to the real azimuth, the real elevation and the speed index for every point of the grid. After this step we are left with three new tables (one for azimuth, one for elevation and one for the speed index), which consists of sets of coefficients for the parabolas, one set for every point of the grid of apparent values. Together with the coefficients of the wind speed, they constitute the final product of the calibration. The tables of coefficients derived from the direction calibration can be indicated as

$$
\begin{aligned}
& \left(C_{a j} \mid a_{\mathrm{app}}, e_{\mathrm{app}}\right) \\
& \left(C_{e j} \mid a_{\mathrm{app}}, e_{\mathrm{app}}\right) \\
& \left(C_{i j} \mid a_{\mathrm{app}}, e_{\mathrm{app}}\right),
\end{aligned}
$$

with $j=1,2,3$. If $N$ is the number of points on the grid of apparent values, the number of data in each table is $3 N$ and the total number of calibration data is $9 N$ for the direction, plus 2 for the velocity. The number of points, $N$, is not fixed beforehand, because the number of grid points of real values need not be the same as $N$, although they will not differ very much. For our range of calibration angles (see section 3 ), $N$ is about 475 .

To find, e.g., the value of the real azimuth at a wind speed $v$ from measured data, we first calculate the apparent azimuth and elevation from the registered signals, which is a straightforward procedure. These values will hardly ever be multiples of $5^{\circ}$, so that we have to make a double linear interpolation (for azimuth and for elevation) between the values at the nearest grid points to get the coefficients. In this way we end up with three coefficients, say $C_{a 1}\left(a_{\mathrm{app}}, e_{\mathrm{app}}\right), C_{a 2}\left(a_{\mathrm{app}}\right.$, $\left.e_{\text {app }}\right)$ and $C_{a 3}\left(a_{\text {app }}, e_{\text {app }}\right)$, and the real azimuth is

$$
\begin{aligned}
a_{r}\left(a_{\mathrm{app}}, e_{\mathrm{app}}\right)= & C_{a 1}\left(a_{\mathrm{app}}, e_{\mathrm{app}}\right) * v^{2} \\
& +C_{a 2}\left(a_{\mathrm{app}}, e_{\mathrm{app}}\right) * v+C_{a 3}\left(a_{\mathrm{app}}, e_{\mathrm{app}}\right) .
\end{aligned}
$$

One problem remains to be solved before we can apply the new method to the interpretation of measured data: the wind speed $v$ is one of the quantities to be determined, but we need it beforehand to be able to apply the tables. Fortunately the changes of the calibration with speed are small, which makes it possible to use an iteration procedure.

Before we start the iteration, we select from the data the maximum signal $s_{m}$ and calculate the apparent azimuth and elevation; this gives us the right grid point in the table of coefficients for the speed index.

The procedure then starts by assuming some value for the wind speed, say $10 \mathrm{~m} \mathrm{~s}^{-1}$. From this value and the table of coefficients we determine the first approximation to the speed index $i_{s}$. The quotient $s_{m} / i_{s}$ is the signal that component $A$ would have had in the same situation, but with the instrument oriented (relative to the wind) as during the wind speed calibration. So, with the aid of the wind speed calibration, we get a new approximation for the wind speed:

$$
v=f\left(s_{m} / i_{s}\right) \text {. }
$$

With this approximation for $v$ we use the coefficients for the speed index again to obtain a new value for $i_{s}$; this value and $s_{m}$ give us the next approximation to the wind speed. The procedure is repeated again until we have a sufficient level of convergence. Usually we need less than three iteration steps to get convergence to the level of $\mathrm{cm} \mathrm{s}^{-1}$.

\section{Application to measurements}

We have performed eight calibrations for our sonic, the Kayo Denki DAT-300 mentioned earlier, during the period 1980 to 1984 (and some as well later on, but these were not subjected to the treatment that follows). Although we did the first calibration more or less as a test, the results were such that we decided to do it on a routine basis. A small advantage (but possibly significant for less attentive experimenters) advantage of our method is that the useful opening angle of the 
TABLE 1. Results of the sonic calibrations with regard to the wind speed.

\begin{tabular}{|c|c|c|c|c|c|c|c|}
\hline \multirow[b]{2}{*}{$\begin{array}{c}\begin{array}{c}\text { Date }^{\dagger} \\
\text { (Month-Yr) }\end{array} \\
\end{array}$} & \multicolumn{3}{|c|}{ A } & \multicolumn{4}{|c|}{$\mathbf{B}^{\dagger \dagger}$} \\
\hline & $\begin{array}{c}\left(U_{1}-U_{p}\right) \\
\left(\mathrm{cm} \mathrm{s}^{-1}\right)\end{array}$ & $\begin{array}{c}\left(U_{t}-U_{x}\right) \\
\left(\mathrm{cm} \mathrm{s}^{-1}\right)\end{array}$ & $\begin{array}{c}\left(U_{t}-U_{f}\right) \\
\left(\mathrm{cm} \mathrm{s}^{-1}\right)\end{array}$ & $\begin{array}{c}Y \\
\left(\mathrm{~cm} \mathrm{~s}^{-1}\right)\end{array}$ & $\begin{array}{c}a z \\
\text { (deg) }\end{array}$ & $\begin{array}{c}Z \\
\left(\mathrm{~cm} \mathrm{~s}^{-1}\right)\end{array}$ & $\begin{array}{c}e l \\
(\mathrm{deg})\end{array}$ \\
\hline $6-80$ & $1 \pm 8$ & $18 \pm 7$ & $2 \pm 19$ & 11 & 1.3 & 5 & 0.6 \\
\hline & & & & 38 & 1.1 & 39 & 1.1 \\
\hline $1-81$ & $1 \pm 10$ & $-83 \pm 51$ & $-89 \pm 45$ & 8 & 0.8 & 1 & 0.1 \\
\hline & & & & 35 & .0 .9 & 68 & 1.8 \\
\hline 6-81 & $-0 \pm 14$ & $-32 \pm 18$ & $-29 \pm 17$ & -4 & -0.4 & 13 & 1.4 \\
\hline & & & & -22 & -0.6 & 69 & 2.1 \\
\hline $2-83$ & $1 \pm 5$ & $-33 \pm 19$ & $-37 \pm 9$ & -10 & -1.1 & 10 & 1.1 \\
\hline & & & & -31 & -0.9 & 47 & 1.3 \\
\hline $5-83$ & $-1 \pm 5$ & $-47 \pm 32$ & $-53 \pm 20$ & 0 & 0.0 & 0 & 0.0 \\
\hline & & & & 3 & 0.1 & 17 & 0.5 \\
\hline $1-84$ & $0 \pm 7$ & $29 \pm 8$ & $30 \pm 8$ & -5 & -0.6 & 9 & 0.9 \\
\hline & & & & 16 & -0.5 & 54 & 1.5 \\
\hline $9-84$ & $0 \pm 15$ & $25 \pm 22$ & $24 \pm 22$ & -8 & -1.0 & 9 & 1.0 \\
\hline & & & & -31 & -0.9 & 53 & 1.6 \\
\hline $12-84$ & $0 \pm 4$ & $23 \pm 13$ & $22 \pm 13$ & -9 & -1.0 & 11 & 1.2 \\
\hline All: & $|0| \pm 10$ & $|30| \pm 20$ & $|30| \pm 20$ & -33 & -1.0 & 59 & 1.7 \\
\hline
\end{tabular}

A: differences between the tunnel speed $\left(U_{t}\right)$ and the wind speed calculated respectively with the calibration parameterization $\left(U_{p}\right)$, from the "X" signal of the sonic $\left(U_{x}\right)$ and from the total signal, assuming a cosine characteristic $\left(U_{f}=\left(X^{2}+Y^{2}+Z^{2}\right)^{1 / 2}\right.$. B: The values presented are averages over the full calibration interval. The $Y$ and $Z$ are the sonic data for the horizontal cross wind and vertical wind speed during the wind speed calibration (in principle along the $X$-axis), $a z=\operatorname{arctg}(Y / X)$ and $e l=\operatorname{arctg}\left[Z /\left(X^{2}+Y^{2}\right)^{1 / 2}\right]$.

${ }^{\dagger}$ The calibrations are indicated by month and year minus 1900 .

${ }^{\mathrm{H}}$ The upper value is the average at a tunnel speed of $5 \mathrm{~m} / \mathrm{s}$, the lower at $20 \mathrm{~m} / \mathrm{s}$.

instrument can be expanded from the usual $90^{\circ}$ to $120^{\circ}$, the azimuth range of our calibration. Table 1 summarizes the results of the wind speed calibrations. The first column indicates the date of the calibration; the next three columns (labeled A) compare results obtained with the new method and those using the cosine assumption, and the final four columns (labeled B) give information about the calibration circumstances. The $U_{t}$ indicates the wind tunnel speed (the reference speed ), $U_{p}$ the wind speed as calculated with our method, $U_{x}$ the wind speed obtained from the $X$ component of the sonic (horizontal, along wind) and $U_{f}$ the wind speed calculated from all signals, assuming a cosine direction characteristic. We have added this last quantity to take care of a possible misalignment of the instrument and its mounting in the wind tunnel; in the case of a perfect alignment and an ideal instrument there should be no differences between the third and fourth column. The figures are the averages over the full calibration range of the indicated differences.

The results are unequivocal: both the bias with respect to the wind tunnel speed and the fluctuations around it are smaller for the $U_{p}$ values than for the other two. The fact that the fluctuations are smaller shows that the differences can not be explained by a zero-offset, which would change the average, but would leave the fluctuations unaffected.

Column 5 of Table 1 shows the wind speeds indicated by the $Y$-component (horizontal, cross wind) at a tunnel speed of $5 \mathrm{~m} \mathrm{~s}^{-1}$ (upper line) and $20 \mathrm{~m} \mathrm{~s}^{-1}$ (lower line) during each wind speed calibration. These values have been converted into azimuth angles in column 6 by combining them with the corresponding $X$ values. The figures in column 6 show a good correspondence between the upper and lower values, indicating that the instrument had indeed the calculated azimuth deviation. The deviations are small (around $1^{\circ}$ in most cases), which is necessary to be able to apply our method: One of the basic assumptions in the procedure for the sonic anemometer is that during the wind speed calibration, the orientation of the instrument is such that only the component selected can sense the wind. Column 7 corresponds to column 5 , but now for the elevation, and column 8 relates in the same way to column 6 . In all cases we see a rise in the values of the elevation if we go from 5 to $20 \mathrm{~m} \mathrm{~s}^{-1}$. This indicates that the instrument and/or its mounting are not completely rigid, but yield somewhat in the airstream. It is possible to compensate for this effect in the data handling, provided one assumes that the effect is not due to a deformation of the instrument itself, but only to a lack of stiffness of the calibration frame. If the sensor head should be deformed by the force of the wind, no correction should be used, as the effect then is a part of the calibration itself.

Figure 2 depicts the variation of the wind speed calibration with time. The figure shows the difference between the wind speed obtained with the calibration indicated on the $x$-axis and the one obtained with the calibration of June 1980. The difference is shown for 


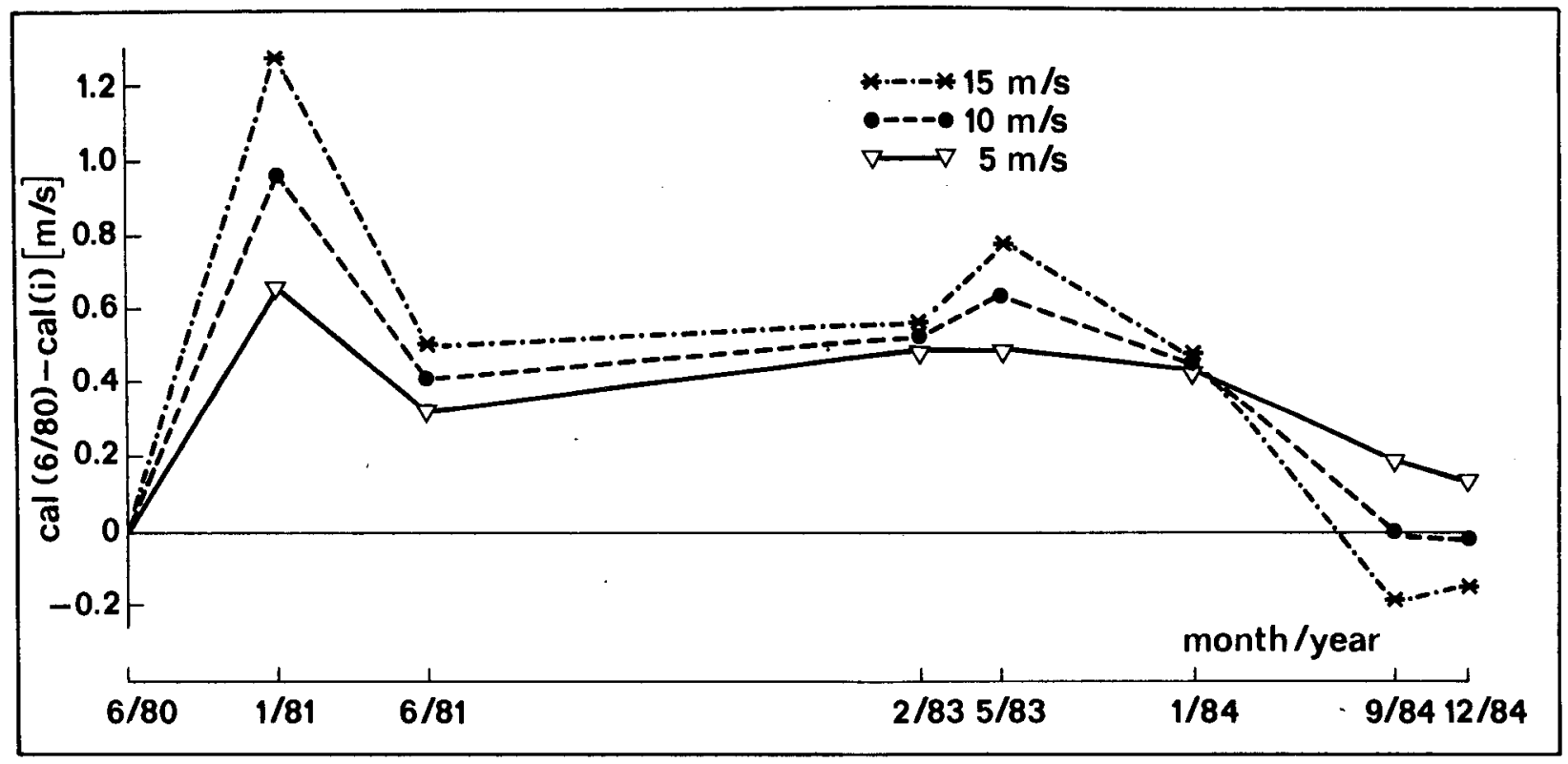

FIG. 2. Variations in the wind speed calibration of a sonic anemometer during the period 1980-1984. Plotted are the differences between the wind speed calculated with the calibration of June $1980(6 / 80)$ and the one calculated with later calibrations. The calculations were performed for three signal levels, corresponding to respectively $5(\nabla), 10(\bullet)$ and $15(*) \mathrm{m} \mathrm{s}^{-1}$, using the $6 / 80$ calibration.

three signal levels, viz., those that correspond to 5,10 and $15 \mathrm{~m} \mathrm{~s}^{-1}$, respectively, for the June 1980 calibration.

So far the information presented is only concerning the speed calibration and the resulting wind speed. To give an indication about the variations resulting from the full calibration, we have processed 5 sets of data, which were obtained in 1980 and for which the calibration of June 1980 applies, with all eight calibrations and a cosine direction dependence as well. The results are given in Figs. 3 and 4. In Fig. 3 we present the results for mean wind speed, azimuth and elevation, in Fig. 4 those for one component of the turbulent kinetic energy $\left(u^{\prime} u^{\prime}\right)$ and for the vertical momentum flux $\left(u^{\prime} w^{\prime}\right)$. All values are $30-\mathrm{min}$ means, and the sampling frequency was $70 \mathrm{~Hz}$. The sets were selected to get good coverage over the whole range of wind speeds and for the constancy of the wind speed over the period. The nominal wind speeds for the 5 sets are $6,8,10$, 12 and $14 \mathrm{~m} \mathrm{~s}^{-1}$. The values presented are again the differences between the values obtained with the calibration indicated along the $x$-axis and those resulting from the June 1980 calibration.

The figures do show some general trends, but not very clear ones. The wind speed, elevation and energy differences go to lower values in the course of time, and the azimuth and momentum values go up. This could be ascribed to an aging process of the sensors, proceeding at different rates for different sensors. On these general trends rather large random fluctuations are superimposed. These may have been caused by small deformations of the frame, e.g., as a result of slight damages during transport.

With regard to the consequences of deformations we would like to remark that in a separate study we have also investigated how they affect sonic temperature readings and found that a change of $1 \mathrm{~mm}$. in the distance between the transducers causes an offset of $3^{\circ} \mathrm{C}$. Furthermore it has been suggested that the sonic anemometer could be used for humidity measurements. In the same study we derive that the effect of deformations is then even more dramatic: a deformation of $1 \mathrm{~mm}$ can cause a humidity error of $100 \%$.

\section{Discussion and conclusions}

The new calibration method produces results which are substantially different from those obtained with the usual assumption of a cosine direction dependence. A comparison under laboratory conditions, i.e., between the calibration results and the readings of the wind speed gauge of the tunnel, gives us the conviction that these results are not only different, but also better. The precision, needed for research applications such as the determination of vertical momentum fluxes by the eddy-correlation method, therefore in our opinion necessitates the use of our method (or some comparable one), despite the complicated procedure.

The use of our calibration method is only meaningful if the errors due to the experimental situation (misalignment of the instrument and disturbance of the airflow by surrounding obstacles) are smaller then or of the same order as our corrections. 


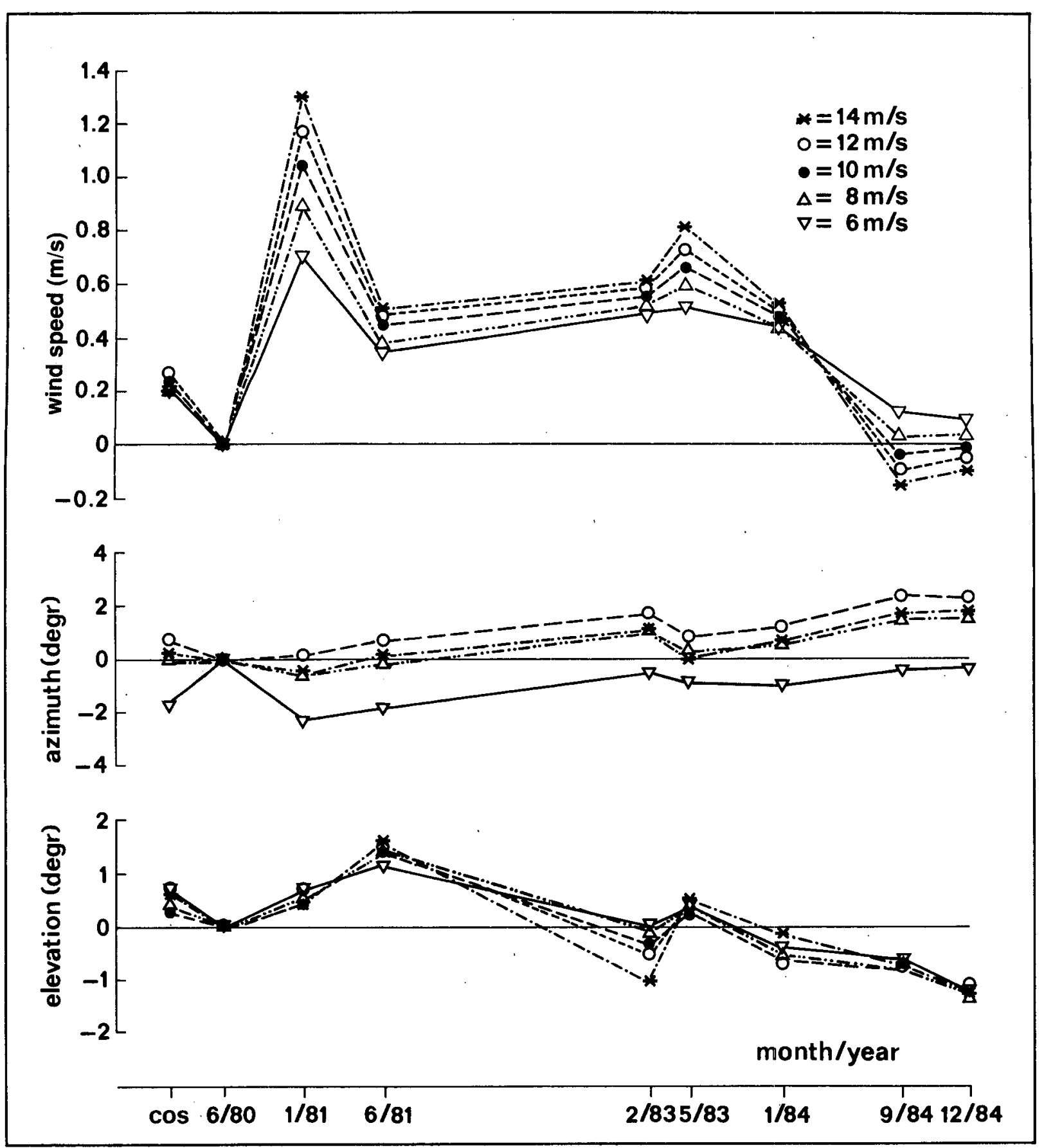

FIG. 3. Variations in mean wind speed, azimuth and elevation during the period 1980-1984. All calibrations were applied to five datasets, with wind speeds between 6 and $14 \mathrm{~m} \mathrm{~s}^{-1}$, which were obtained in an experiment performed in 1980 and for which the $6 / 80$ calibration is valid. Plotted are the differences between the values resulting from the application of the indicated calibration and those using the correct one. The term "cos" indicates the result assuming a cosine direction dependence.

We have tried to achieve this in our own experiments, at a research platform in the Dutch coastal waters, by the following measures:

- We used a $20-\mathrm{m}$ boom, reaching out horizontally from the platform. This was an adequate length to be free from most of the flow distortion due to the platform body, as has been shown in a wind tunnel study on a model of the platform.

- The construction that the instruments were mounted on at the end of the boom was made as slender as possible. Although the model by Wyngaard 


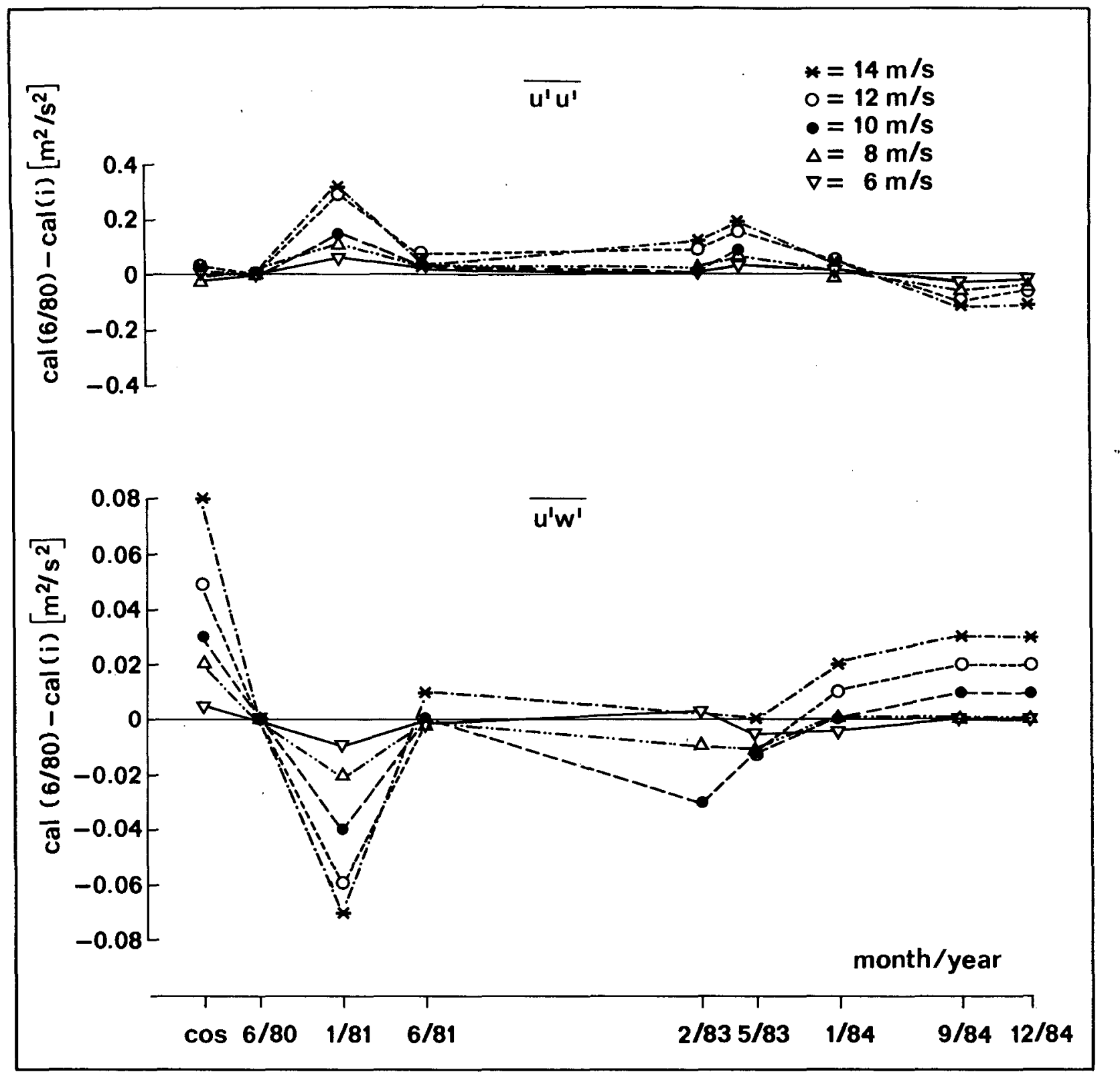

FIG. 4. As in Fig. 3, but for a component of the turbulent kinetic energy $\left(u^{\prime} u^{\prime}\right)$ and the vertical momentum flux $\left(u^{\prime} w^{\prime}\right)$.

(1981) offers in principle a possibility to calculate the remaining corrections, its application is not straightforward, as the construction has no simple geometric shape. A better approach would have been calibration of the anemometer on the end construction; for practical reasons (the size of the measuring section of the wind tunnel) we had to abandon this refinement, which should certainly be practiced where feasible.

- We have furthermore taken much care over the mounting of the instrument in order to know its precise orientation with respect to the boom. During an ex- periment the position of the boom was then continuously recorded with two mutually orthogonal electronic levels (accuracy $0.1^{\circ}$ ). Incorporating these levels in the anemometer would have simplified the procedure, but was not done because the position information was also necessary for other instruments used.

- Finally, the bending of the instrument by the force of the wind is already included in the calibration, as indicated earlier.

The trends in and fluctuations of the results, shown in Figs. 2, 3 and 4, indicate the need for frequent cal- 
ibrations. Since we discovered this, we made it our habit to calibrate before and after a field phase. The small differences between these calibrations sets have led us to believe that this is sufficient to prevent the publication of doubtful values.

As indicated already, the method is not restricted to a specific type of wind meter, but is-in principle at least-applicable to any anemometer without moving parts. For example, the pressure anemometer mentioned earlier, has in its present configuration a double sine direction dependence (a full sine curve over a range of $180^{\circ}$ ) and is calibrated in the same way as the sonic, over an azimuth range of $180^{\circ}$.

As a final remark, we want to indicate that the way in which we have used the $x$ - and $y$-axis of the DAT 300 sonic are the reverse of the indications in the manufacturer's handbook. Our way is in conformity with usual meteorological practice.

Acknowledgments. We want to thank Mr. E. Worrell and Mr. J. Schaap who performed the actual calibra- tions and Mr. E. Wiggers, who assisted us with the calculations.

\section{REFERENCES}

Fox, H. L., 1968: Continuous wave three-component sonic anemometer. Final Rep., AFCRL-68-0180, 76 pp.

Geernaert, G. L., S. E. Larsen and F. Hansen, 1987: Measurements of the wind stress, heat flux, and turbulence intensity during storm conditions over the North Sea. J. Geophys. Res., 92, 13127-13139.

Jones, E. P., and S. D. Smith, 1978: The air density correction to eddy flux measurements. Bound.-Layer Meteor., 15, 357-360.

Oost, W. A., 1983: The pressure anemometer-an instrument for adverse circumstances. J. Climate Appl. Meteor., 22, 2075-2084.

Wucknitz, J., 1980: Flow distortion by supporting structures. AirSea Interaction, Instruments and Methods F. Dobson, L. Hasse and R. Davis, Eds., Plenum Press, 605-626.

Wyngaard, J. C., 1981: The effects of probe-induced flow distortion on atmospheric turbulence measurements. J. Appl. Meteor., 20, 784-794.

-_ , and Shi-Feng Zhang, 1985: Transducer-shadow effects on turbulence spectra measured by sonic anemometers. J. Atmos. Oceanic Technol., 2, 548-558. 